\title{
Coulomb interaction effects in spin-polarized transport
}

\author{
Irene D'Amico \\ Istituto Nazionale per la Fisica della Materia (INFM), \\ and Institute for Scientific Interchange (ISI), Viale Settimio Severo 65, I-10133 Torino, Italy \\ Giovanni Vignale \\ Department of Physics, University of Missouri, Columbia, Missouri 65211
}

(Received 14 August 2001; revised manuscript received 19 October 2001; published 8 February 2002)

\begin{abstract}
We study the effect of the electron-electron interaction on the transport of spin-polarized currents in metals and doped semiconductors in the diffusive regime. In addition to well-known screening effects, we identify two additional effects, which depend on many-body correlations and exchange and reduce the spin-diffusion constant. The first is the "spin Coulomb drag"-an intrinsic friction mechanism which operates whenever the average velocities of up-spin and down-spin electrons differ. The second arises from the decrease in the longitudinal spin stiffness of an interacting electron gas relative to a noninteracting one. Both effects are studied in detail for both degenerate and nondegenerate carriers in metals and semiconductors, and various limiting cases are worked out analytically. The behavior of the spin-diffusion constant at and below a ferromagnetic transition temperature is also discussed.
\end{abstract}

DOI: 10.1103/PhysRevB.65.085109

PACS number(s): 72.25. $-\mathrm{b}, 75.40 . \mathrm{Gb}, 85.75 .-\mathrm{d}$

\section{INTRODUCTION}

The theory of spin-polarized transport is attracting the attention of the physics community both for its potential applications to the emerging field of "spintronics" 1 and in relation to the intriguing field of quantum computation. ${ }^{2}$ In this context Kikkawa et al. $^{3,4}$ generated much excitement by demonstrating optical injection and subsequent control of packets of spin polarization in the conduction band of $n$-doped GaAs. Such packets could in principle be used to transport information between separate regions of a system. Unlike ordinary electron-hole packets, whose mobility is limited by strong scattering in the hole component, these unipolar electronic spin packets are both long lived (with lifetime $\tau_{s} \gtrsim 10 \mathrm{~ns}$ ) and highly mobile. ${ }^{4}$

From the theoretical point of view, most recent work on spin-transport phenomena is based on an independent electron model, where, in general, the coupling between different spin channels is completely neglected. Flatté and Byers ${ }^{5}$ have recently discussed the behavior of spin packets in semiconductors in the framework of the Hartree approximation, where the Coulomb interaction is taken into account only through the imposition of a charge neutrality constraint. This constraint establishes an electrostatic coupling between different spin channels. They neglect, however, all further many-body effects.

In this paper we go beyond the treatment by Flatté and Byers by considering more subtle correlation (spin-drag) and exchange (spin-softening) effects. Our objective is the derivation of drift-diffusion equations for spin packets in a full many-body context, i.e., allowing for correlation between different spin channels. These equations contain the spinpacket mobility and the diffusion constant as key parameters. We show that both exchange interactions and the drag effect between different spin populations ${ }^{6}$ concur in reducing the value of the diffusion constant by a sizable amount, leaving at the same time the mobility basically unaffected. The mi- croscopic quantities responsible for this effect are the longitudinal spin stiffness (the second derivative of the free energy with respect to magnetization, not to be confused with the transverse spin stiffness of the ferromagnetic state) and the spin-drag transresistivity. We discuss in detail the behavior of these quantities in various regimes and show how important the Coulomb effects are when dealing with doped semiconductors (see Sec. III).

This paper is organized as follows. In Sec. II we review the basic ideas underlying the spin-resolved drift-diffusion equations and make use of the Landau transport equation to elucidate the structure of the homogeneous spin resistivity matrix. In particular, we show that the off-diagonal element of the spin-resistivity matrix (the spin transresistivity) is almost exclusively controlled by the Coulomb interaction: the contribution from spin-flip scattering, while finite, is utterly negligible for short-range scatterers.

In Sec. III we present the calculation of the Coulomb contribution to the spin trans-resistivity. The theory of Ref. 6 is extended in various directions. First we study the spindrag effect as a function of temperature going from the degenerate regime (which is appropriate for ordinary metals) to the nondegenerate regime, which is appropriate for lowdensity/high-temperature doped semiconductors. Then, we calculate the spin drag in the "mixed" case in which one spin component is degenerate while the other is nondegenerate: this is relevant to situations in which a strong spin polarization exists.

Section IV is devoted to a description of the behavior of the longitudinal spin stiffness of the homogeneous electron gas as a function of density and temperature.

In Sec. $\mathrm{V}$ we present a detailed derivation of the driftdiffusion equation for a macroscopic spin packet that extend the results of Ref. 7. We give explicit expressions for the mobility and diffusion constant in terms of microscopic quantities such as the spin stiffness and the spin transresistivity discussing in detail the general case in which the mo- 
mentum relaxation rates for the two spin populations may differ. Section VI suggests experiments aimed at directly measuring the spin transresistivity and the spin-diffusion constant in metals or semiconductors.

Electron-gas theory predicts that, at sufficiently low temperatures and densities, the homogeneous electron gas undergoes a ferromagnetic transition. ${ }^{8,9}$ Such a transition could be, in principle, observed in a doped semiconductor. Section VII examines the behavior of spin-diffusion constant in the paramagnetic phase and how the occurrence of ferromagnetism as a second-order phase transition would affect it. Our calculations show that the diffusion constant vanishes at the transition temperature and increases as the system becomes fully spin polarized with decreasing temperature.

\section{GENERAL THEORY I}

\section{A. Drift-diffusion equation}

The theory of diffusive transport in metals and semiconductors is based on the assumption of a local linear relationship between the current densities of up- and down-spin electrons and the gradient of the local electrochemical potentials,

$$
-e \vec{J}_{\alpha}(\vec{r})=-\sum_{\beta} \sigma_{\alpha \beta}(\vec{r}) \vec{\nabla} \psi_{\beta}(\vec{r}) .
$$

Here $e$ is the absolute value of the electron charge, $\vec{J}_{\alpha}(\vec{r})(\alpha=\uparrow$ or $\downarrow)$ are number currents, ${ }^{10}$ and the electrochemical potentials $\psi_{\alpha}(\vec{r})$ are given by the electrostatic potential $\phi(\vec{r})$ plus the local chemical potential, which can be spin dependent,

$$
\psi_{\alpha}(\vec{r})=\phi(\vec{r})-\frac{1}{e} \frac{\partial f\left(n_{\uparrow}, n_{\downarrow}, T\right)}{\partial n_{\alpha}} .
$$

Here $f\left(n_{\uparrow}, n_{\downarrow}, T\right)$ is the free energy per unit volume of a homogeneous interacting electron gas evaluated at the local spin densities $n_{\alpha}(\vec{r})$ and uniform temperature $T ;{ }^{11} \sigma_{\alpha \beta}$ $\equiv \lim _{\omega \rightarrow 0} \sigma_{\alpha \beta}(q=0, \omega)$ is the homogeneous conductivity matrix of the electron gas, whose structure, in the presence of interactions, will be elucidated below.

Substituting Eq. (2) in Eq. (1) and writing

$$
\vec{\nabla} \frac{\partial f\left(n_{\uparrow}, n_{\downarrow}, T\right)}{\partial n_{\alpha}}=\sum_{\beta} \frac{\partial^{2} f\left(n_{\uparrow}, n_{\downarrow}, T\right)}{\partial n_{\alpha} \partial n_{\beta}} \vec{\nabla} n_{\beta},
$$

we obtain

$$
e \vec{J}_{\alpha}(\vec{r})=\sum_{\beta}\left[\sigma_{\alpha \beta}(\vec{r}) \vec{\nabla} \phi(\vec{r})-e D_{\alpha \beta}(\vec{r}) \vec{\nabla} n_{\beta}(\vec{r})\right],
$$

where the diffusion matrix $D_{\alpha \beta}$ is given by

$$
e^{2} D_{\alpha \beta}=\sum_{\gamma} \sigma_{\alpha \gamma} S_{\gamma \beta}
$$

and

$$
S_{\alpha \beta}=\frac{\partial^{2} f\left(n_{\uparrow}, n_{\downarrow}, T\right)}{\partial n_{\alpha} \partial n_{\beta}}
$$

is the static longitudinal spin-stiffness matrix-the inverse of the spin-susceptibility matrix $\chi_{\alpha \beta}$. Equation (5) is the wellknown Einstein relation between the mobility and the diffusion constant, generalized here to the case of spin-polarized transport. The first term of Eq. (4) is the drift current associated with the electrostatic field, the second is the diffusion current associated with the gradient of the electronic densities. These two terms cancel out exactly in a situation of local equilibrium, due to the Einstein relation and the fact that $\left[\chi^{-1}\right]_{\alpha \beta}=S_{\alpha \beta}$.

On a formal level the main effect of the Coulomb interaction is the appearance of nonvanishing off-diagonal elements of the conductivity and spin-stiffness matrices. $\sigma_{\uparrow \downarrow}$ $\neq 0$ implies that an electric field acting only on the up-spin electrons must necessarily drag along a current of down-spin electrons. Conversely, a current of up-spin electrons $\left(J_{\uparrow}\right.$ $\neq 0)$ flowing against a background of stationary down-spin electrons $\left(J_{\downarrow}=0\right)$ will necessarily induce a gradient of spindown electrochemical potential $\vec{E}_{\downarrow}=-\nabla \psi_{\downarrow}=e \rho_{\downarrow} \vec{J}_{\uparrow}$, where $\rho_{\alpha \beta}$ is the resistivity matrix, inverse to $\sigma_{\alpha \beta}$. We shall see later how one can make use of these effects for a direct experimental measure of the spin Coulomb drag.

The other noteworthy feature is $S_{\uparrow \downarrow} \neq 0$ : this means that the chemical potential of up spins $\partial f\left(n_{\uparrow}, n_{\downarrow}, T\right) / \partial n_{\uparrow}$ is a function of both up and down spin densities. Thus a disturbance acting on one of the two spin populations will affect the other through Coulomb correlation.

\section{B. Structure of the resistivity matrix}

Although the homogeneous resistivity matrix can be calculated from first-principle Kubo formulas and/or transport equations its general structure (including the off-diagonal terms, due mostly to the Coulomb interaction) is best understood at the phenomenological level. We first present the phenomenological picture and then justify it from a more formal consideration of the Landau transport equation.

Let $\vec{E}_{\uparrow}(t)$ and $\vec{E}_{\downarrow}(t)$ be uniform effective electric fields, $\vec{E}_{\alpha}=-\nabla \psi_{\alpha}$, that couple to up and down spins, respectively. We restrict ourselves to the linear-response regime. If $\vec{v}_{\alpha}$ is the velocity of the center of mass of electrons of spin $\alpha$, and $N_{\alpha}$ the number of such electrons, then the equation of motion for $\vec{v}_{\alpha}$ has the form

$$
m^{*} N_{\alpha} \dot{\vec{v}}_{\alpha}=-e N_{\alpha} \vec{E}_{\alpha}+\vec{F}_{\alpha \bar{\alpha}}^{C}-\frac{m^{*}}{\tau_{\alpha}} N_{\alpha} \vec{v}_{\alpha}+\frac{m^{*}}{\tau_{\alpha}^{\prime}} N_{\alpha}^{-} \vec{v}_{\bar{\alpha}},
$$

where $m^{*}$ is the effective mass of the carriers and $\bar{\alpha} \equiv-\alpha$.

Let us examine the meaning of the various terms in Eq. (7). The first term on the right-hand side is the net force exerted by the electric field on spin- $\alpha$ electrons.

The second term, $\vec{F}_{\alpha \bar{\alpha}}^{C}$, is the Coulomb force exerted by spins of the opposite orientation $\bar{\alpha}$ on spin- $\alpha$ electrons. Notice that the net force exerted by spins of the same orientation vanishes by virtue of Newton's third law. For exactly the same reason we must have $\vec{F}_{\alpha \bar{\alpha}}^{C}=-\vec{F}_{\alpha \alpha}^{C}$, and by Galilean 
invariance this force can only depend on the relative velocity of the two components. Hence, in the linear approximation, we can write

$$
\vec{F}_{\alpha \bar{\alpha}}^{C}=-\gamma m^{*} N_{\alpha} \frac{n_{\alpha}^{-}}{n}\left(\vec{v}_{\alpha}-\vec{v}_{\alpha}^{-}\right),
$$

where $n=n_{\uparrow}+n_{\downarrow}$ is the total density. Equation (8) defines the spin-drag coefficient $\gamma$.

The third term on the right-hand side of Eq. (7) combines two distinct physical effects. One is the net force exerted on spin- $\alpha$ electrons by electron-impurity collisions that $d o$ not flip the spin of the incoming electrons. The other is the rate at which momentum is lost to the $\alpha$ component as a result of electron-impurity collisions that flip the spin from $\alpha$ to $\bar{\alpha}$. Accordingly, the momentum relaxation rate $\tau_{\alpha}^{-1}$ is written as the sum of non-spin-flip and spin-flip contributions: $\tau_{\alpha}^{-1}$ $=\tau_{n f, \alpha}^{-1}+\tau_{s f, \alpha}^{-1}$. Spin-flip times have been found to be very long both in metals $\left[\tau_{s f, \alpha} \sim 1 \mathrm{~ns}\right.$ (Ref. 12)] and in semiconductors $\left[\tau_{s f, \alpha} \sim 10 \mathrm{~ns}\right.$ (Refs. 3 and 4)], whereas non-spin-flip times are usually much shorter (of the order $10^{-3}-10^{-4} \mathrm{~ns}$ ). Thus, for most practical purposes, $\tau_{\alpha}^{-1} \approx \tau_{n f, \alpha}^{-1}$.

Finally, the last term on the right-hand side of Eq. (7) represents the rate at which momentum is fed into the $\alpha$ component by electron-impurity collisions that flip the spin from $\bar{\alpha}$ to $\alpha$. Because electrons emerging from collisions have a randomized momentum distribution with nearly zero average, we expect $\left(\tau_{\alpha}^{\prime}\right)^{-1}$ to be smaller than even the already small spin-flip rate $\tau_{s f, \alpha}^{-1}$. Indeed, we shall see in the next section that $\left(\tau_{\alpha}^{\prime}\right)^{-1}$ vanishes for short-range scatterers in the Born approximation.

Fourier transforming the equation of motion (7) for the current density $\vec{j}_{\alpha}(\omega)=-e n_{\alpha} \vec{v}_{\alpha}(\omega)$, we find

$$
\begin{aligned}
i \omega \vec{j}_{\alpha}(\omega)= & -\frac{n_{\alpha} e^{2}}{m^{*}} \vec{E}_{\alpha}(\omega)+\left(\frac{n_{\alpha}^{-}}{n} \gamma+\frac{1}{\tau_{\alpha}}\right) \vec{j}_{\alpha}(\omega) \\
& -\left(\frac{n_{\alpha}}{n} \gamma+\frac{1}{\tau_{\alpha}^{\prime}}\right) \vec{j}_{\bar{\alpha}}(\omega) .
\end{aligned}
$$

The resistivity matrix $\rho_{\alpha \alpha^{\prime}}$ is defined as the coefficient of proportionality between the electric field and the current, i.e., $\vec{E}_{\alpha}=\Sigma_{\alpha^{\prime}} \rho_{\alpha \alpha^{\prime}} \vec{j}_{\alpha^{\prime}}$. A quick comparison between this definition and Eq. (9) shows that the complete form of the resistivity matrix $\rho_{\alpha \beta}$ is

$$
\rho=\left(\begin{array}{cc}
-i \omega \frac{m^{*}}{e^{2} n_{\uparrow}}+\frac{m^{*}}{n_{\uparrow} e^{2} \tau_{\uparrow}}+\frac{n_{\downarrow}}{n_{\uparrow}} \frac{m^{*}}{n e^{2}} \gamma & -\frac{m^{*}}{n_{\uparrow} e^{2} \tau_{\uparrow}^{\prime}}-\frac{m^{*}}{n e^{2}} \gamma \\
-\frac{m^{*}}{n_{\downarrow} e^{2} \tau_{\downarrow}^{\prime}}-\frac{m^{*}}{n e^{2}} \gamma & -i \omega \frac{m^{*}}{e^{2} n_{\downarrow}}+\frac{m^{*}}{n_{\downarrow} e^{2} \tau_{\downarrow}}+\frac{n_{\uparrow}}{n_{\downarrow}} \frac{m^{*}}{n e^{2}} \gamma
\end{array}\right) .
$$

Notice that this matrix is symmetric, due to the relation $1 / n_{\downarrow} \tau_{\downarrow}^{\prime}=1 / n_{\uparrow} \tau_{\uparrow}^{\prime}$, which will be proved in the next section.

Due to the extreme smallness of the spin-flip rates $1 / \tau_{\alpha}^{\prime}$, the off-diagonal resistivity (also referred to as "spin transresistivity") is controlled almost entirely by the Coulomb interaction term, i.e., we can safely assume

$$
\rho_{\uparrow \downarrow}=-\frac{m^{*}}{n e^{2}} \gamma
$$

and $\gamma$ is directly proportional to the spin transresistivity. But then, Galilean invariance and Newton's third law demand that the same $\rho_{\uparrow \downarrow}$ appear also as a correction to the ordinary diagonal resistivity. This effect is quite distinct from the "trivial" renormalizations due to the electronic screening of these interactions.

\section{Derivation from Landau transport equation}

In this section we provide a microscopic justification of the phenomenological Eq. (7) and give explicit expressions for the electron-impurity relaxation rates. We start from the linearized transport equation for the quasiparticle distribution function in Landau theory of Fermi liquid. ${ }^{13}$ This is simply the classical Boltzmann equation for quasiparticles in a selfconsistent field described by Landau parameters. In the homogeneous case the self-consistent field coincides with the classical electric field and the distribution function $f_{\alpha}(\vec{k}, t)$ obeys the well-known kinetic equation

$$
\frac{\partial f_{\alpha}(\vec{k}, t)}{\partial t}-e \vec{E}_{\alpha} \cdot \frac{\partial f_{\alpha}^{(0)}(\vec{k})}{\partial \vec{k}}=\left(\frac{\partial f_{\alpha}(\vec{k}, t)}{\partial t}\right)_{\text {coll }},
$$

where $f_{\alpha}^{(0)}(\vec{k})$ is the equilibrium distribution function.

The collision term $\left[\partial f_{\alpha}(\vec{k}, t) / \partial t\right]_{\text {coll }}$ has contributions from the Coulomb interaction as well as spin-flip and nonspin-flip electron-impurity interactions. The various contributions are listed below.

Coulomb collisions:

$$
\begin{aligned}
\left(\frac{\partial f_{\alpha}(\vec{k}, t)}{\partial t}\right)_{\text {coll }}^{C}= & -\sum_{\vec{p} \vec{k}^{\prime} p^{\prime}, \beta \alpha^{\prime} \beta^{\prime}} W^{C}\left(\vec{k} \alpha, \vec{p} \beta ; \vec{k}^{\prime} \alpha^{\prime}, \vec{p}^{\prime} \beta^{\prime}\right) \\
& \times \delta_{\vec{k}+\vec{p}, \vec{k}^{\prime}+\vec{p}^{\prime}} \delta_{\alpha+\beta, \alpha^{\prime}+\beta^{\prime}}\left\{f_{\alpha}(\vec{k}) f_{\beta}(\vec{p})\right. \\
& \times\left[1-f_{\alpha^{\prime}}\left(\vec{k}^{\prime}\right)\right]\left[1-f_{\beta^{\prime}}\left(\vec{p}^{\prime}\right)\right]-\left[1-f_{\alpha}(\vec{k})\right]
\end{aligned}
$$




$$
\begin{aligned}
& \left.\times\left[1-f_{\beta}(\vec{p})\right] f_{\alpha^{\prime}}\left(\vec{k}^{\prime}\right) f_{\beta^{\prime}}\left(\vec{p}^{\prime}\right)\right\} \\
& \times \delta\left(\epsilon_{k \alpha}+\epsilon_{p \beta^{\prime}}-\epsilon_{k^{\prime}, \beta^{\prime}}-\epsilon_{p^{\prime} \beta^{\prime}}\right),
\end{aligned}
$$

where $W^{C}\left(\vec{k} \alpha, \vec{p} \beta ; \vec{k}^{\prime} \alpha^{\prime}, \vec{p}^{\prime} \beta^{\prime}\right)$ is the probability of the Coulomb scattering process $\vec{k} \alpha, \vec{p} \beta \rightarrow \vec{k}^{\prime} \alpha^{\prime}, \vec{p}^{\prime} \beta^{\prime}$ and $\epsilon_{k \alpha}$ is the energy of a particle of momentum $\vec{k}$ and spin $\alpha$ relative to the chemical potential. The conservations of momentum, energy, and spin are explicitly displayed.

Non-spin-flip electron-impurity collisions:

$$
\begin{aligned}
\left(\frac{\partial f_{\alpha}(\vec{k}, t)}{\partial t}\right)_{\text {coll }}^{n f}= & -\sum_{\vec{k}^{\prime}} W^{n f}\left(\vec{k} \alpha, \vec{k}^{\prime} \alpha\right)\left[f_{\alpha}(\vec{k})-f_{\alpha}\left(\vec{k}^{\prime}\right)\right] \\
& \times \delta\left(\epsilon_{k \alpha}-\epsilon_{k^{\prime} \alpha}\right),
\end{aligned}
$$

where $W^{n f}\left(\vec{k} \alpha, \vec{k}^{\prime} \alpha\right)$ is the probability of the non-spin-flip (nf) scattering process $\vec{k} \alpha \rightarrow \vec{k}^{\prime} \alpha$.

Spin-flip electron-impurity collisions:

$$
\begin{aligned}
\left(\frac{\partial f_{\alpha}(\vec{k}, t)}{\partial t}\right)_{\text {coll }}^{s f}= & -\sum_{\vec{k}^{\prime}} W^{s f}\left(\vec{k} \alpha, \vec{k}^{\prime} \bar{\alpha}\right)\left[f_{\alpha}(\vec{k})-f_{\bar{\alpha}}\left(\vec{k}^{\prime}\right)\right] \\
& \times \delta\left(\epsilon_{k \alpha}-\epsilon_{k^{\prime}} \bar{\alpha}\right),
\end{aligned}
$$

where $W^{s f}\left(\vec{k} \alpha, \vec{k}^{\prime} \bar{\alpha}\right)$ is the probability of the spin-flip (sf) scattering process $\vec{k} \alpha \rightarrow \vec{k}^{\prime} \bar{\alpha}$.

In order to obtain a closed equation of motion for the currents, such as Eq. (9), we must multiply both sides of Eq. (12) by $-e \vec{k} / m^{*}$, sum over $\vec{k}$, and then express the integrated collision term

$$
\vec{F}_{\alpha} \equiv \sum_{\vec{k}} \vec{k}\left(\frac{\partial f_{\alpha}(\vec{k}, t)}{\partial t}\right)_{\text {coll }}
$$

back in terms of the currents. Of course, this cannot be done rigorously, but for an isotropic system slightly perturbed from equilibrium one can assume ${ }^{14}$ that the distribution function of the state with currents $\vec{j}_{\alpha}=-e n_{\alpha} \vec{v}_{\alpha}$ is given by

$$
f_{\alpha}(\vec{k}, t)=f_{\alpha}^{(0)}\left(\epsilon_{k \alpha}\right)-\frac{\partial f_{\alpha}^{(0)}\left(\epsilon_{k \alpha}\right)}{\partial \epsilon_{k \alpha}} \vec{v}_{\alpha}(t) \cdot \vec{k}
$$

Substituting this into Eqs. (13)-(15), and linearizing with respect to the currents wherever needed, we arrive, after tedious but straightforward manipulations, at the desired equation of motion (9), with the following expressions for the various relaxation times:

$$
\begin{aligned}
\gamma= & \frac{n}{m^{*} N_{\alpha} n_{\bar{\alpha}}} \sum_{\vec{k} p \vec{k}^{\prime} \vec{p}^{\prime}} \frac{\left(\vec{k}-\vec{k}^{\prime}\right)^{2}}{2 d k_{B} T} \\
& \times W^{C}\left(\vec{k} \alpha, \vec{p} \bar{\alpha} ; \vec{k}^{\prime} \alpha, \vec{p}^{\prime} \bar{\alpha}\right) \delta_{\vec{k}+\vec{p}, \vec{k}^{\prime}+\vec{p}^{\prime}} \\
& \times \delta\left(\epsilon_{k \alpha}+\epsilon_{p \bar{\alpha}}-\epsilon_{k^{\prime}, \alpha}-\epsilon_{p^{\prime}}-\bar{\alpha}\right) f_{\alpha}^{(0)}\left(\epsilon_{k \alpha}\right) \\
& \times f_{\bar{\alpha}}^{(0)}\left(\epsilon_{p}\right) f_{\alpha}^{(0)}\left(-\epsilon_{k^{\prime} \alpha}\right) f_{\alpha}^{(0)}\left(-\epsilon_{p^{\prime}}\right),
\end{aligned}
$$

where $d$ is the number of spatial dimensions;

$$
\begin{aligned}
\frac{1}{\tau_{n f, \alpha}}= & -\sum_{\vec{k}} \frac{\partial f_{\alpha}^{(0)}\left(\epsilon_{k \alpha}\right)}{\partial \epsilon_{k \alpha}} \frac{k^{2}}{n_{\alpha} d} \sum_{\vec{k}^{\prime}} W^{n f}\left(\vec{k} \alpha, \vec{k}^{\prime} \alpha\right) \\
& \times\left(1-\hat{k} \cdot \hat{k}^{\prime}\right) \delta\left(\epsilon_{k \alpha}-\epsilon_{k^{\prime} \alpha}\right),
\end{aligned}
$$

where $\hat{k}$ and $\hat{k}^{\prime}$ are unit vectors in the directions of $\vec{k}$ and $\vec{k}^{\prime}$;

$$
\begin{aligned}
\frac{1}{\tau_{s f, \alpha}}= & -\sum_{\vec{k}} \frac{\partial f_{\alpha}^{(0)}\left(\epsilon_{k \alpha}\right)}{\partial \epsilon_{k \alpha}} \frac{k^{2}}{n_{\alpha} d} \sum_{\vec{k}^{\prime}} W^{s f}\left(\vec{k} \alpha, \vec{k}^{\prime} \bar{\alpha}\right) \\
& \times \delta\left(\epsilon_{k \alpha}-\epsilon_{k^{\prime}} \bar{\alpha}\right), \\
\frac{1}{\tau_{\alpha}^{\prime}}= & -\frac{1}{n_{\bar{\alpha}} d} \sum_{\vec{k}} \frac{\partial f_{\alpha}^{(0)}\left(\epsilon_{k \alpha}\right)}{\partial \epsilon_{k \alpha}} \sum_{\vec{k}^{\prime}} W^{s f}\left(\vec{k} \alpha, \vec{k}^{\prime} \bar{\alpha}\right) \vec{k} \cdot \vec{k}^{\prime} \\
& \times \delta\left(\epsilon_{k \alpha}-\epsilon_{k^{\prime}}-\bar{\alpha}\right) .
\end{aligned}
$$

Notice that $1 / \tau_{s f, \alpha}$ and $1 / \tau_{\alpha}^{\prime}$ arise, respectively, from the first and the second term on the right side of Eq. (15). The key difference between these two relaxation rates is that the expression for the latter involves an angular average of the scattering probability with weight factor $\hat{k} \cdot \hat{k}^{\prime}=\cos (\theta)$. This average vanishes in the Born approximation for short-range scatterers, since the scattering probability becomes isotropic (independent of $\vec{k}$ and $\vec{k}^{\prime}$ ) in this special case. Quite generally, one can expect $1 / \tau_{\alpha}^{\prime}$ to be much smaller than $1 / \tau_{s f, \alpha}$ in agreement with the qualitative arguments given in the previous section. This means that the spin transresistivity is almost entirely a Coulomb interaction effect, and therefore its measurement can shed light on the nature of the Coulomb correlation between up- and down-spin electrons. This is one of the main points we wanted to make in this section.

Finally, notice that Eq. (21) implies the identity

$$
\frac{1}{n_{\downarrow} \tau_{\downarrow}^{\prime}}=\frac{1}{n_{\uparrow} \tau_{\uparrow}^{\prime}}
$$

which guarantees the symmetry of the resistivity matrix Eq. (10).

\section{CALCULATION OF THE SPIN TRANSRESISTIVITY}

The theory of the spin transresistivity has been worked out in Ref. 6. This theory closely parallels the theory of the ordinary Coulomb drag between parallel two-dimensional electron or hole-gas layers ${ }^{15}$ but differs in some important details, as the fact that electrons of opposite spin interact with the same set of impurities, so that certain electronimpurity terms which appear in the Kubo formulation of the transresistivity do not vanish upon disorder averaging. Fortunately, it turned out that these terms cancel out exactly at low frequency $\left(\omega \ll E_{F}\right)$ and to leading order in the electronelectron and electron-impurity interactions. ${ }^{6}$

The final outcome of Ref. 6 was that the spintransresistivity, for $\omega=0$, is given by 


$$
\begin{aligned}
\rho_{\uparrow \downarrow}(T)= & \frac{\beta}{n_{\uparrow} n_{\downarrow} e^{2}} \frac{1}{V} \sum_{\vec{q}} \frac{q^{2}}{3} v_{q}^{2} \frac{1}{2} \\
& \times \int_{0}^{\infty} \frac{d \omega^{\prime}}{\pi} \frac{\chi_{0 \uparrow}^{\prime \prime}\left(q, \omega^{\prime}\right) \chi_{0 \downarrow}^{\prime \prime}\left(q,-\omega^{\prime}\right)}{\left|\varepsilon\left(q, \omega^{\prime}\right)\right|^{2} \sinh ^{2}\left(\beta \omega^{\prime} / 2\right)},
\end{aligned}
$$

where $\beta=1 / k_{B} T, \quad k_{B}$ is the Boltzmann constant, $v_{q}$ $=4 \pi e^{2} / q^{2} \epsilon$ is the Fourier transform of the Coulomb interaction with $\epsilon$ the dielectric constant of the material, $V$ is the volume of the system, $\chi_{0 \alpha}(q, \omega)$ is the noninteracting spinresolved density-density response function, and $\varepsilon(q, \omega)=1$ $-v_{q} \chi_{0 \uparrow}(\vec{q}, \omega)-v_{q} \chi_{0 \downarrow}(\vec{q}, \omega)$ is the random-phase approximation (RPA) dielectric function. This expression, which is based on an approximate decoupling of a four-point response function (generalized RPA, see Ref. 6), is valid in the weak Coulomb and impurity scattering regime, characterized by $\hbar / \tau_{D} \ll k_{B} T$, where $\tau_{D}=\left(n_{\uparrow} / n\right) \tau_{\uparrow}+\left(n_{\downarrow} / n\right) \tau_{\downarrow}$. Because of the "high" temperature, weak-localization effects are negligible.

Equation (23) will be our starting point: at variance with the calculation of Ref. 6 we present our results not only in the low-temperature limit (which is relevant to metals and where $\gamma \sim T^{2}$ ), but also in the nondegenerate $k_{B} T \gg E_{F}$ and quasidegenerate $k_{B} T \sim E_{F}$ regimes (which are relevant to doped semiconductors), where $E_{F}=\hbar^{2}\left(3 \pi^{2} n\right)^{2 / 3} / 2 m *$ is the Fermi energy. ${ }^{16}$

\section{A. Numerical evaluation}

To calculate $\rho_{\uparrow \downarrow}$ at finite temperature, we have used in Eq. (23) the temperature-dependent expression for the threedimensional noninteracting spin-resolved density-density response function

$$
\begin{aligned}
\chi_{0 \alpha}^{\prime \prime}(q, \omega ; T)= & -\frac{1}{16 \pi} \frac{1}{\bar{q} a^{* 3} R y}\left\{\bar{\omega}-\frac{1}{\beta R y}\right. \\
& \left.\times \ln \frac{1+e^{\beta\left\{\left(1 / \epsilon_{q}\right)\left[\left(\hbar \omega+\epsilon_{q}\right) / 2\right]^{2}-\xi_{\alpha}\right\}}}{1+e^{\beta\left\{\left(1 / \epsilon_{q}\right)\left[\left(\hbar \omega-\epsilon_{q}\right) / 2\right]^{2}-\xi_{\alpha}\right\}}}\right\},
\end{aligned}
$$

where $a^{*}$ is the effective Bohr radius, $\bar{q}=q a^{*}, \bar{\omega}$ $=\hbar \omega / R y, R y=e^{2} / 2 a^{*}$ is the effective Rydberg, $\xi_{\alpha}$ is the chemical potential for the $\alpha$ spin population, and $\epsilon_{q}$ $=\hbar^{2} q^{2} / 2 m^{*}$. Equation (24) follows directly from the definition

$$
\chi_{0 \alpha}^{\prime \prime}(q, \omega ; T)=-\frac{\pi}{V} \sum_{\vec{k}}\left(n_{\vec{k} \alpha}-n_{\vec{k}+\vec{q} \alpha}\right) \delta\left(\hbar \omega+\epsilon_{k}-\epsilon_{k+q}\right),
$$

where $n_{q \alpha}=1 /\left\{\exp \left(\beta\left(\epsilon_{q}-\xi_{\alpha}\right)\right)+1\right\}$ is the average number of $\alpha$-spin electrons with energy $\epsilon_{q}$.

Figure 1 shows $\left|\rho_{\uparrow \downarrow}\right|$ as a function of temperature and density. ${ }^{17}$ The data are calculated in the paramagnetic phase and for semiconductor parameters (GaAs), i.e., $m^{*}=0.067$, $\epsilon=12$, and carrier density $n_{1}=1.5 \times 10^{18} \mathrm{~cm}^{-3}, n_{2}=1.5$ $\times 10^{17} \mathrm{~cm}^{-3}$, and $n_{3}=1.5 \times 10^{16} \mathrm{~cm}^{-3} \cdot \rho_{\uparrow \downarrow}$ peaks at about the Fermi temperature $T_{F}$, underlying the crossing between

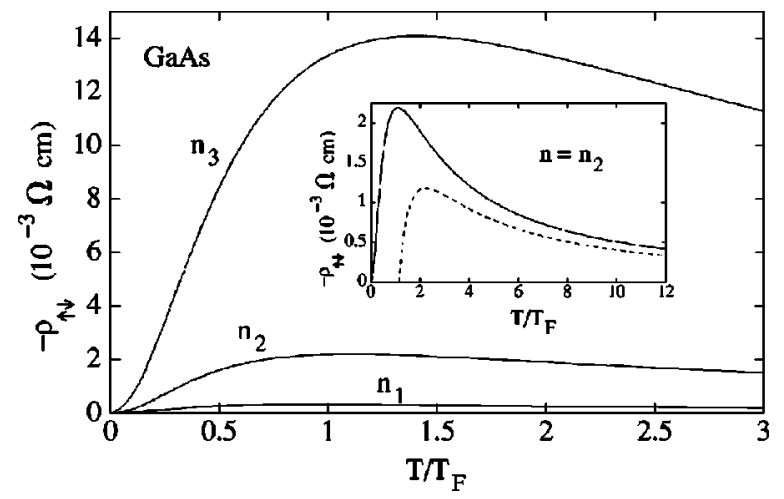

FIG. 1. Spin transresistivity $\rho_{\uparrow \downarrow}$ as a function of temperature (rescaled by $\left.T_{F}\right)$ for GaAs parameters $\left(m^{*}=0.067 m_{e}, \epsilon=12, \mu\right.$ $=3 \times 10^{3} \mathrm{~cm}^{2} / \mathrm{V} \mathrm{s}$ ). Each curve corresponds to a different density: $n_{1}=1.5 \times 10^{18} \mathrm{~cm}^{-3}, \quad n_{2}=1.5 \times 10^{17} \mathrm{~cm}^{-3}, \quad n_{3}=1.5 \times 10^{16} \mathrm{~cm}^{-3}$. Inset: comparison between $\rho_{\uparrow \downarrow}$ and its analytical approximation in the nondegenerate regime vs temperature (rescaled by $T_{F}$ ) for $n$ $=n_{2}$.

the degenerate and the nondegenerate regimes. As can be seen, $\rho_{\uparrow \downarrow}$ is strongly enhanced as the density decreases, mainly due to the prefactor dependence $\sim 1 / n^{2}$. In fact its maximum increases of almost two orders of magnitude, passing from $0.3 \mathrm{~m} \Omega \mathrm{cm}$ for $n=n_{1}$ to $14 \mathrm{~m} \Omega \mathrm{cm}$ for $n=n_{3}$. In the calculations of the following sections, we will mainly focus on the density value $n=n_{2}$, corresponding to a Fermi temperature $T_{F}=178 \mathrm{~K}$. The inset of Fig. 1 presents for this density value the comparison between $\rho_{\uparrow \downarrow}$ and its nondegenerate analytical approximation (dashed lines) discussed in detail in Secs. III C.

We now turn to a quantitative assessment of the relevancy of the spin Coulomb drag. First of all it is necessary to underline that the spin drag is an intrinsic effect of spinpolarized transport: that is, while impurity scattering could in principle be suppressed in a perfect crystal, the spin Coulomb drag will always be present, even in the purest sample, and dominate over phonon scattering at sufficiently low temperature. However, since available samples are usually far from perfection, it is reasonable to ask how the spin transresistivity compares to the more familiar Drude resistivity. In metals, as we shall show in detail in the next section, one finds, at most, $\rho_{\uparrow \downarrow} \sim 10^{-2} \mu \Omega \mathrm{cm}$ so that $\rho_{\uparrow \downarrow} / \rho_{D}$ is of the order of few percent. The situation is very different for semiconductors: since both the Fermi temperature (at which $\rho_{\uparrow \downarrow}$ peaks) and the carrier density are considerably lower than in metals, $\rho_{\uparrow \downarrow}$ can become comparable and even greater than $\rho_{D}$. This strong variation depends on specific semiconductor characteristics, such as the effective mass, mobility, and density of the carriers.

In Fig. 2 we show the effect of the carrier mobility $\mu$ on the ratio $\rho_{\uparrow \downarrow} / \rho_{D}$. We plot $\rho_{\uparrow \downarrow} / \rho_{D}$ in respect to temperature for an $n$-doped semiconductor as GaAs $\left(m^{*}=0.067 m_{e}, \epsilon\right.$ $=12$, and $n=1.5 \times 10^{17} \mathrm{~cm}^{-3}$; upper panel) and for a $p$-doped semiconductor, as $(\mathrm{Ga}, \mathrm{Mn}) \mathrm{As} \quad\left(m^{*}=0.5 m_{e}, \boldsymbol{\epsilon}\right.$ $=12, n=1.2 \times 10^{19} \mathrm{~cm}^{-3}$; lower panel). Each curve corresponds to a different mobility value as reported in the figure caption. The values increase from "A" to "D." In particular 

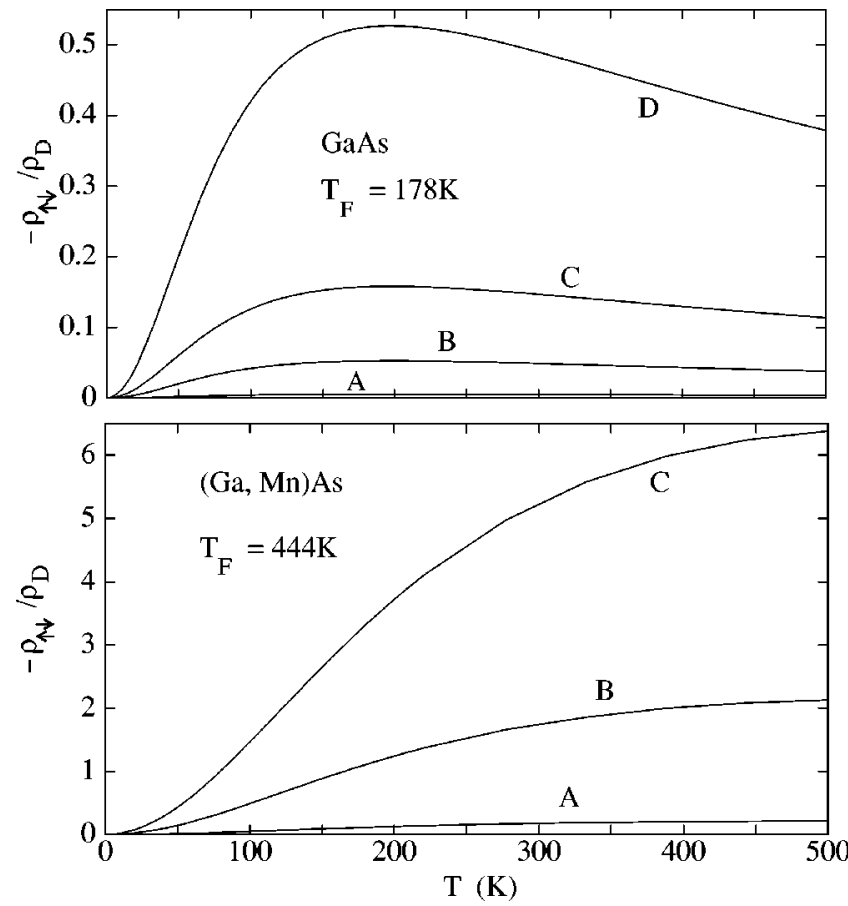

FIG. 2. Upper panel: $\rho_{\uparrow \downarrow} / \rho_{D}$ as a function of temperature for GaAs parameters $\left(m^{*}=0.067 m_{e}, \quad \epsilon=12, \quad n=1.5 \times 10^{17} \mathrm{~cm}^{-3}\right)$. Each curve corresponds to a different mobility: $A=10^{2} \mathrm{~cm}^{2} / \mathrm{V} \mathrm{s}$, $B=10^{3} \mathrm{~cm}^{2} / \mathrm{V} \mathrm{s}, \quad C=3 \times 10^{3} \mathrm{~cm}^{2} / \mathrm{V} \mathrm{s}, \quad D=10^{4} \mathrm{~cm}^{2} / \mathrm{V} \mathrm{s}$, as labeled. Lower panel: same as upper panel but for $(\mathrm{Ga}, \mathrm{Mn}) \mathrm{As}$ parameters $\left(m^{*}=0.5 m_{e}, \epsilon=12, n=1.2 \times 10^{19} \mathrm{~cm}^{-3}\right)$.

the value $\mu=3 \times 10^{3} \mathrm{~cm}^{2} / \mathrm{V} \mathrm{s}$ (labeled as "C"), corresponds to the value measured for a spin packet in Ref. 4. As can be seen, changing the material it is possible to increase the ratio $\rho_{\uparrow \downarrow} / \rho_{D}$ by an order of magnitude, to the point that the spin transresistivity can become greater than the Drude resistivity.

In Fig. 3 we show the dependence on the ratio $\rho_{\uparrow \downarrow} / \rho_{D}$ on the carrier density for GaAs. The results are presented for two different temperatures $(T=20 \mathrm{~K}$, dashed lines and $T$ $=300 \mathrm{~K}$, solid line) and two different values of the mobility

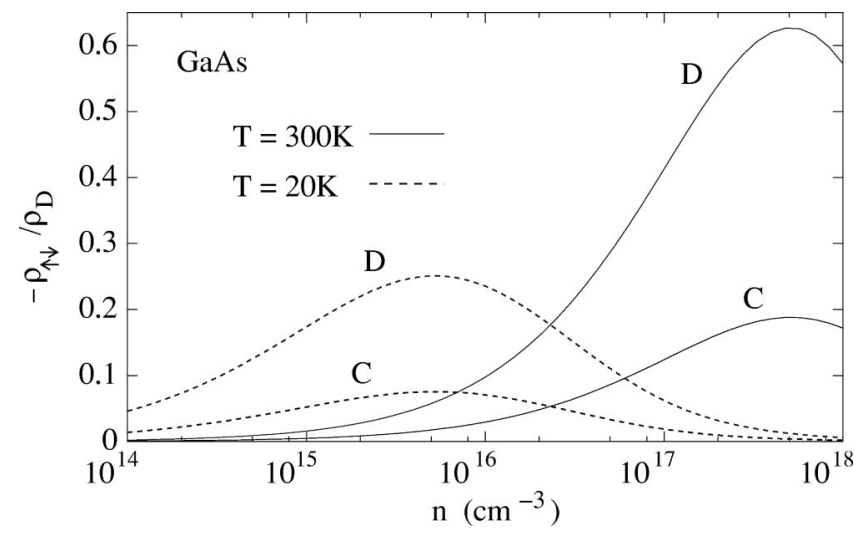

FIG. 3. $\rho_{\uparrow \downarrow} / \rho_{D}$ as a function of carrier density for GaAs parameters $\left(m^{*}=0.067 m_{e}, \epsilon=12\right)$. The solid curves are calculated at the temperature $T=300 \mathrm{~K}$, while the dashed curves at $T=20 \mathrm{~K}$. For each temperature two different mobilities are considered, $C=3$ $\times 10^{3} \mathrm{~cm}^{2} / \mathrm{V} \mathrm{s}$ and $D=10^{4} \mathrm{~cm}^{2} / \mathrm{V} \mathrm{s}$, as labeled. (as labeled in the figure). Each curve peaks at a density such that $T_{F}=T$.

In conclusion, our calculations demonstrate that in semiconductors, at $T \approx T_{F}$, the spin Coulomb drag must definitely be considered an important contribution to the resistivity for spin polarized currents.

\section{B. Degenerate limit}

The only regime of practical importance in ordinary highdensity metals is the low-temperature $\left(k_{B} T \ll E_{F}\right)$ and lowfrequency $\left(\hbar \omega \ll E_{F}\right)$ one. In this regime the spin-Coulomb drag coefficient is controlled by a subset of all the processes that lead to the finite lifetime of a quasiparticle at the Fermi surface, namely, the processes in which the quasiparticle in question exchanges momentum with an electron of opposite spin (scattering processes between parallel spin electrons do not cause relaxation of the spin current). Since the inverse quasiparticle lifetime at the Fermi surface is known in Fermiliquid theory to scale as $\left(k_{B} T / E_{F}\right)^{2}$ we expect the same scaling to hold for the spin drag coefficient at low temperature. This expectation is confirmed by the detailed calculation, ${ }^{6}$ which, at $\omega=0$ gives

$$
\begin{aligned}
\rho_{\uparrow \downarrow}(T)= & -\frac{\hbar a^{*}}{e^{2}} \frac{2 \pi^{2}\left(k_{B} T\right)^{2}}{3(R y)^{2}} \cdot \frac{1}{24 \pi^{3} \bar{n}_{\downarrow} \bar{n}_{\uparrow}} \\
& \times \int_{0}^{2 k_{F} a^{*}} \frac{d \bar{q}}{\bar{q}^{2}} \frac{1}{\left|\varepsilon\left(\bar{q} / a^{*}, 0\right)\right|^{2}},
\end{aligned}
$$

where $k_{F} \equiv \min \left(k_{F \uparrow}, k_{F \downarrow}\right)$, with $k_{F \alpha}$ the $\alpha$ spin population Fermi wave vector and $\bar{n}_{\alpha} \equiv n_{\alpha} a^{* 3}$. Equation (26) shows that, in the absence of impurities, $\rho_{\uparrow \downarrow}(T)$ is proportional to $T^{2}$. This dependence is not affected by modifications in the form of $\chi_{0 \alpha}(q, \omega)$ due to the presence of impurities. ${ }^{6}$ For $r_{s} \gtrsim 5, r_{s}$ the usual electron-gas parameter, the spin transresistivity is appreciable $\left[\left|\rho_{\uparrow \downarrow}(T \gtrsim 40 \mathrm{~K})\right| \gtrsim 0.01 \mu \Omega \mathrm{cm}^{6}\right]$. For $r_{s}=5$, the Coulomb scattering time from Eq. (11) is $\gamma^{-1}$ $\approx 10^{-13}$ s while $\delta_{s} / v_{F} \approx 10^{-10} \mathrm{~s}$ where $\delta_{s}$ is the spin relaxation length: $\gamma^{-1}$ is several orders of magnitude smaller than the spin-flip time, confirming that neglecting spin-flip processes is indeed a good approximation for this kind of metal.

\section{Nondegenerate limit}

The nondegenerate limit is characterized by $T \gg T_{F}$. First of all we calculate the nondegenerate limit of the noninteracting temperature-dependent spectral function Eq. (24): starting from the definition Eq. (25), we use the classical expression for the fugacity $\exp \left(\beta \xi_{\alpha}\right)=n_{\alpha} \cdot 8 \pi^{3}\left(\beta / 2 m^{*} \pi\right)^{3 / 2}$, and obtain

$$
\begin{aligned}
\chi_{0 \alpha}^{\prime \prime}(q, \omega ; T)= & -\frac{\sqrt{2 \pi \beta m^{*}} n_{\alpha}}{\hbar q} \exp \left(-\frac{\beta \epsilon_{q}}{4}\right) \\
& \times \exp \left(-\frac{\beta \hbar^{2} \omega^{2}}{4 \epsilon_{q}}\right) \sinh \left(\frac{\beta \hbar \omega}{2}\right) .
\end{aligned}
$$

In addition, in order to calculate the nondegenerate limit $\rho_{\uparrow \downarrow}$, we have used the classical limit for the dielectric constant $\varepsilon(q, \omega)=1+\left(4 \pi e^{2} / \epsilon q^{2}\right)\left(n / k_{B} T\right)$. The final result is 


$$
\begin{gathered}
\rho_{\uparrow \downarrow}(T)=\frac{8 e^{2} \sqrt{m^{*}}}{\sqrt{2 \pi^{3}}\left(k_{B} T\right)^{3 / 2} \epsilon^{2}} \int_{0}^{\infty} d x \frac{x \exp (-x)}{(x+\lambda)^{2}} \\
\approx \frac{8 e^{2} \sqrt{m^{*}}}{\sqrt{2 \pi^{3}}\left(k_{B} T\right)^{3 / 2} \epsilon^{2}}[-1-C-\ln (\lambda)],
\end{gathered}
$$

where the second expression, Eq. (29), is valid in the limit $\lambda \ll 1, \lambda=\hbar^{2} k_{D}^{2} / k_{B} T 4 m^{*}, k_{D}^{2} \equiv 4 \pi e^{2} n / \epsilon k_{B} T$ is the inverse of the squared Debye screening length and $C \approx 0.577$ is the Euler's constant. Notice that, in the nondegenerate limit, $\rho_{\uparrow \downarrow}$ becomes almost independent of the total density $n$ and independent of the spin-density components $n_{\alpha}$, while a quantum-mechanical dependence on $\hbar$ survives even in this regime. $\rho_{\uparrow \downarrow}$ tends to zero as $\left(k_{B} T\right)^{-3 / 2} \ln \left(k_{B} T\right)$ as $T \rightarrow \infty$. The inset of Fig. 1 illustrates the comparison between $\rho_{\uparrow \downarrow}$ and its asymptotic form. This approximation becomes valid for $T$ $\gg T_{F}$, but, since $T_{F} \sim n^{2 / 3}$, such limit is fulfilled only at very low carrier densities.

\section{Mixed (degenerate/nondegenerate) case}

A very interesting limit is the one corresponding to a spinpolarization process, for which $n_{\uparrow} \rightarrow n$ and $n_{\downarrow} \rightarrow 0$. This is indeed relevant for one of the problems we want to analyze, i.e., a semiconductor with strongly spin-polarized carriers. This system is in a very peculiar state: its minority downspin population is nondegenerate, i.e., $k_{B} T \gg E_{F \downarrow}, E_{F \alpha}$ $=\hbar^{2}\left(6 \pi^{2} n_{\alpha}\right)^{2 / 3} / 2 m^{*}$, while, for low enough temperatures, the majority up-spin population is degenerate, i.e., $k_{B} T$ $\ll E_{F \uparrow}$. The expression for the noninteracting spin-resolved density-density response functions entering the spin transresistivity Eq. (23) can then be taken from the previous subsections and are given by

$$
\begin{aligned}
\chi_{0 \uparrow}^{\prime \prime}\left(q, \omega^{\prime} ; T\right)= & -\frac{m^{* 2}}{4 \pi \hbar^{3}} \frac{\omega^{\prime}}{q} \text { for } 0<q<2 k_{F \uparrow}, \\
= & 0 \quad \text { otherwise }
\end{aligned}
$$

and

$$
\chi_{0 \downarrow}^{\prime \prime}\left(q, \omega^{\prime} ; T\right)=-\left(\frac{\pi m^{*}}{2}\right)^{1 / 2} \beta^{3 / 2} n_{\downarrow} \exp \left(-\frac{\beta m^{*} \omega^{\prime 2}}{2 q^{2}}\right) \frac{\omega^{\prime}}{q},
$$

where Eq. (30) is valid up to first order in $\omega^{\prime}$ and Eq. (32) represents the classical limit $(\hbar \rightarrow 0)$ of Eq. (27). Using Eqs. (30)-(32), and the approximation, due to the small density of down-spin carriers $\varepsilon\left(q, \omega^{\prime}\right) \approx \varepsilon_{\uparrow}(q, 0)$, the expression for the spin transresistivity becomes

$$
\begin{aligned}
\rho_{\uparrow \downarrow}(T)= & -\frac{\hbar a^{*}}{e^{2}} \frac{2 \sqrt{\pi}}{9} \frac{\left(k_{B} T\right)^{1 / 2}}{(R y)^{1 / 2}} \frac{1}{\bar{n}_{\uparrow}} \\
& \times \int_{0}^{2 k_{F \uparrow} a^{*}} \frac{d \bar{q}}{\bar{q}^{2}} \frac{1}{\left|\varepsilon_{\uparrow}\left(\bar{q} / a^{*}, 0\right)\right|^{2}} .
\end{aligned}
$$

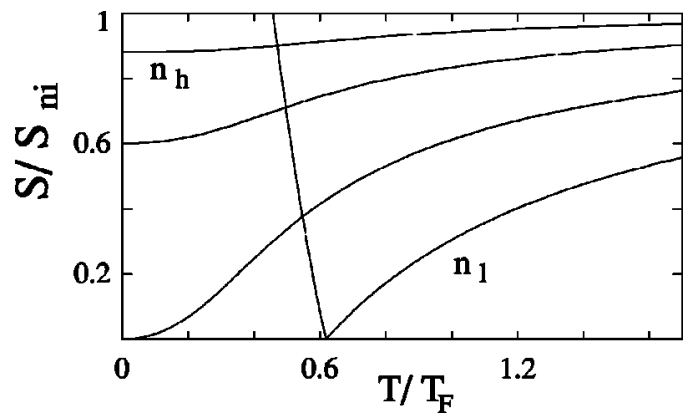

FIG. 4. Spin stiffness $S$, rescaled by its noninteracting approximation $S_{n i}$ vs $T / T_{F}$ and for different carrier densities. The lowest carrier density is $n_{l}=4.2 \times 10^{11} \mathrm{~cm}^{-3}$. The density increases by a factor 100 for each curve up to the value $n_{h}=4.2 \times 10^{17} \mathrm{~cm}^{-3}$ for the upper curve. The cusp corresponds to the onset of ferromagnetism.

Equation (33) is very similar to Eq. (26), the result obtained when both components are degenerate. This is a consequence of the fact that, in the appropriate regime, the nondegenerate spectral function Eq. (32) presents the same dependence in $\omega^{\prime}$ and $q$ as the degenerate one. We want to underline that, in this limit, $\rho_{\uparrow \downarrow}(T)$ is independent of $n_{\downarrow}$ and that in any case $\rho_{\uparrow \downarrow} \rightarrow 0$ for $T \rightarrow 0$.

\section{SPIN STIFFNESS OF AN INTERACTING SPIN-POLARIZED ELECTRON GAS}

The other ingredient entering the drift-diffusion expression for the current, Eq. (4), is the longitudinal spin stiffness matrix $S_{\alpha \beta}$. In particular we are interested in the combination $S=\partial^{2} f(n, m, T) / \partial m^{2}=\left(S_{\uparrow \uparrow}-S_{\uparrow \downarrow}+S_{\downarrow \downarrow}-S_{\downarrow \uparrow}\right) / 4$, where $m=n_{\uparrow}-n_{\downarrow}$, which gives the curvature of the free energy with respect to the magnetization at constant density. This quantity coincides with the inverse of the longitudinal spin susceptibility of the uniform electron gas.

We evaluated $S$ numerically starting from the formulas provided by Tanaka and Ichimaru, ${ }^{18}$ who calculated the freeenergy density of the three-dimensional electron gas as a function of temperature, density, and spin polarization.

Figure 4 shows $S$ divided by its noninteracting value $S_{n i}$ as a function of the dimensionless temperature $T / T_{F}$ for various densities, starting with $n=4.2 \times 10^{17} \mathrm{~cm}^{-3}$ for the upper curve (labeled $n_{h}$ ) down to $n=4.2 \times 10^{11} \mathrm{~cm}^{-3}$ for the lowest one (labeled $n_{l}$ ), decreasing by two orders of magnitude from one curve to the next.

Two regimes are clearly visible. For densities larger than a critical value $n_{c} \simeq 4.2 \times 10^{13} \mathrm{~cm}^{-3}$ the spin stiffness decreases monotonically with decreasing temperature settling to a finite value in the ground state (top two curves). For densities lower than $n_{c}$ a second-order ferromagnetic transition occurs: the critical temperature $T_{c}$ raises from $\sim 0$ at $n$ $=n_{c}$ to a sizeable fraction of the Fermi temperature at $n$ $=4.2 \times 10^{11} \mathrm{~cm}^{-3}$. As in any second-order transition, the spin stiffness vanishes at the transition temperature. ${ }^{19}$ For $T$ $<T_{c}$ the spontaneous magnetization $\bar{m}$ is given by the stable minimum of the free energy, which satisfies the conditions 


$$
\begin{gathered}
\left.\frac{\partial f(n, m, T)}{\partial m}\right|_{m=\bar{m}}=0, \\
\bar{S}=\left.\frac{\partial^{2} f(n, m, T)}{\partial m^{2}}\right|_{m=\bar{m}}>0 .
\end{gathered}
$$

Obviously, ferromagnetism shows up only at extremely low densities, and the electron-gas model may break down well before getting to such densities: it is nevertheless instructive to study the repercussions of the behavior of $S$ on the spindiffusion constant both above and below the critical density.

Let us now consider the limiting behaviors of the spin stiffness at high $\left(T \gg T_{F}\right)$ and low $\left(T \ll T_{F}\right)$ temperatures.

\section{A. High-temperature limit}

In the high-temperature limit $\left[T \gg T_{F}(n)\right]$ the free-energy density of the electron gas has the following expansion:

$$
\begin{aligned}
f\left(n_{\uparrow}, n_{\downarrow}, T\right) \simeq & k_{B} T \sum_{\alpha} n_{\alpha}\left[\ln \left(n_{\alpha} \lambda_{T}^{3}\right)-1\right] \\
& +\frac{k_{B} T}{2^{3 / 2}} \sum_{\alpha} n_{\alpha}^{2} \lambda_{T}^{3}-2 \pi^{2} e^{2} \lambda_{T}^{2} \\
& \times \sum_{\alpha} n_{\alpha}^{2}-\frac{2 \pi^{1 / 2}}{3} \frac{e^{3} n^{3 / 2}}{\left(k_{B} T\right)^{1 / 2}},
\end{aligned}
$$

where $\lambda_{T} \equiv\left(2 \pi \hbar^{2} / m^{*} k_{B} T\right)^{1 / 2}$ is the thermal wavelength. The first term is the free energy of the classical ideal gas, the second term is the leading quantum correction for noninteracting Fermions; ${ }^{20}$ the third term is the leading quantum/ interaction correction, namely, the high-temperature exchange free energy, ${ }^{21}$ the last term is the leading classical interaction correction from Debye-Huckel theory. Only the first three terms depend on the magnetization, and therefore contribute to the spin stiffness. Taking a second derivative with respect to magnetization we find, after simple calculations,

$$
\frac{S}{S_{c}}=1+\frac{n_{\uparrow} n_{\downarrow}}{n^{2}}\left[\frac{n \lambda_{T}^{3}}{2^{1 / 2}}-\frac{8 \pi^{2} e^{2} n^{1 / 3}}{k_{B} T}\left(n \lambda_{T}^{3}\right)^{2 / 3}\right],
$$

where $S_{c}$ is the Curie spin stiffness of an ideal classical gas of density $n$ :

$$
S_{c}=\frac{k_{B} T n}{4 n_{\uparrow} n_{\downarrow}} .
$$

Notice that the leading interaction correction to the noninteracting spin stiffness is negative, in agreement with the behavior seen in Fig. 4.

\section{B. Low-temperature limit}

Let us now examine what happens in the limit $T \rightarrow 0$. Above the critical density $n_{c}$ the spin stiffness simply tends to a constant zero-temperature limit, smaller than the noninteracting value, in agreement with the Landau theory of Fermi liquids.

For $n<n_{c}$ the low-temperature phase is ferromagnetic, and in this case the density of majority spin electrons $\left(n_{\uparrow}\right)$ approaches the total density, while the density of minority spin electrons $\left(n_{\downarrow}\right)$ tends to zero for $T \rightarrow 0$. To understand the behavior of the spin stiffness shown in Fig. 4 we assume that, in the nearly $100 \%$ polarized limit the free energy can be written as the sum of the ground-state energy of the degenerate interacting up-spin gas plus the free energy of an infinitely dilute noninteracting down spin gas:

$$
f\left(n_{\uparrow}, n_{\downarrow}, T\right) \simeq \epsilon_{0}\left(n_{\uparrow}\right)-k_{B} T n_{\downarrow} \ln (1-\zeta)+\text { const },
$$

where $\epsilon_{0}(n)$ is the ground-state energy density of a degenerate liquid of up-spin electrons, and $\zeta=\left(n_{\uparrow}-n_{\downarrow}\right) /\left(n_{\uparrow}\right.$ $\left.+n_{\downarrow}\right)$ is the degree of spin polarization. Obviously, this approximation ignores the correlation between down- and upspin electrons, or, more precisely, presumes that this correlation is smaller than the entropic term $k_{B} T n_{\downarrow} \ln (1-\zeta)$ for $\zeta$ $\rightarrow 1$.

Starting from Eq. (38) it is trivial to show that the minority spin density vanishes for $T \rightarrow 0$ as

$$
n_{\downarrow} \sim n e^{\epsilon_{0}^{\prime}(n) / k_{B} T},
$$

where $\epsilon_{0}^{\prime}(n)=d \epsilon_{0}(n) / d n<0$ at low density, while the spin stiffness goes as

$$
S \sim \frac{k_{B} T}{4 n_{\downarrow}},
$$

which diverges exponentially for $T \rightarrow 0$. This is precisely what our numerical calculations, based on the formulas of Ref. 18, indicate. As we shall see, this result is important in understanding the behavior of the diffusion constant of a unipolar spin packet when the system is fully spin polarized.

\section{EVOLUTION OF A SPIN PACKET}

We will now examine the motion of a spin packet under the effect of a uniform electric field. We are going to generalize the derivation sketched in Ref. 7 discussing also the subtleties involved in the implementation of the charge neutrality constraint. At variance with Ref. 7, we will focus on the general case of different scattering times for the two spin populations. Additionally we will underline how familiar concepts such as the relationship between mobility and conductivity or the Einstein relationship are modified by including Coulomb interaction between different spin populations.

We are going to describe the time evolution of a spin packet obtained by injecting an excess spin density near the origin at time $t=0$. To solve this problem we start from the generalized continuity equations for the spin-density components,

$$
\frac{\partial \Delta n_{\alpha}(\vec{r}, t)}{\partial t}=-\frac{\Delta n_{\alpha}(\vec{r}, t)}{\tau_{s f, \alpha}}+\frac{\Delta n_{\alpha}^{-}(\vec{r}, t)}{\tau_{s f, \bar{\alpha}}}-\vec{\nabla} \cdot \vec{J}_{\alpha}(\vec{r}),
$$


where $\Delta n_{\alpha}(\vec{r}, t) \equiv n_{\alpha}(\vec{r}, t)-n_{\alpha}^{(0)}, \quad n_{\alpha}^{(0)}$ is the equilibrium value of the $\alpha$-density component and $\tau_{s f, \alpha}$ is its spin-flip relaxation time. Substituting in this equation the driftdiffusion expression for the current Eq. (4), we obtain the two equations

$$
\begin{aligned}
\frac{\partial \Delta n_{\alpha}(\vec{r}, t)}{\partial t}= & -\frac{\Delta n_{\alpha}(\vec{r}, t)}{\tau_{s f, \alpha}}+\frac{\Delta n_{\alpha}(\vec{r}, t)}{\tau_{s f, \bar{\alpha}}} \\
& +\frac{E}{e} \sum_{\beta} \frac{\partial \tilde{\sigma}_{\alpha}}{\partial n_{\beta}} \nabla\left(\Delta n_{\beta}\right)+\tilde{\sigma}_{\alpha} \frac{\nabla \cdot E}{e} \\
& +\sum_{\beta}\left[\nabla D_{\alpha \beta} \nabla n_{\beta}+D_{\alpha \beta} \nabla^{2}\left(\Delta n_{\beta}\right)\right],
\end{aligned}
$$

$(\alpha=\uparrow$ or $\downarrow$ ) where

$$
\tilde{\sigma}_{\alpha}=\sum_{\beta} \sigma_{\alpha \beta} .
$$

Equation (42) includes the term $\tilde{\sigma}_{\alpha} \nabla \cdot E / e=\tilde{\sigma}_{\alpha}(\Delta n$ $\left.+\Delta n_{\downarrow}\right) / \epsilon$. This term is "dangerous" because it contains the product of a large quantity $\tilde{\sigma}_{\alpha}$ times a small quantity, the space charge $\Delta n_{\uparrow}+\Delta n_{\downarrow}$, the product itself being of the order of the other quantities of interest in the calculation.

It is tempting, but wrong, to invoke the local charge neutrality constraint

$$
\Delta n_{\uparrow}(r)=-\Delta n_{\downarrow}(r)
$$

at this point. Instead, we will first combine the two components of Eqs. (42) to eliminate the space charge $(\nabla \cdot E)$ term, and only after doing that we can impose, without serious loss of accuracy, the charge neutrality constraint. To eliminate the $\nabla \cdot E$ term we multiply each component of Eq. (42) by the conductivity $\tilde{\sigma}_{\alpha}^{-}$of the opposite channel, and, in order to get the equation of motion for the excess spin density, we subtract the equation for $\alpha$ spins from the equation for $\bar{\alpha}$ spins. Only at this point we impose the local charge neutrality constraint Eq. (44). With this procedure we obtain the correct drift-diffusion equation

$$
\begin{aligned}
\frac{\partial \Delta m(\vec{r}, t)}{\partial t}= & -\frac{\Delta m(\vec{r}, t)}{\tau_{s}}+\frac{\sum_{\alpha} \tilde{\sigma}_{\alpha}^{-} \nabla\left[\tilde{D}_{\alpha} \nabla \Delta m(\vec{r}, t)\right]}{\sum_{\alpha} \tilde{\sigma}_{\alpha}} \\
& +\frac{\sum_{\alpha} \tilde{\sigma}_{\alpha}^{-} \tilde{\mu}_{\alpha}}{\sum_{\alpha} \tilde{\sigma}_{\alpha}} \vec{E} \cdot \vec{\nabla} \Delta m(\vec{r}, t),
\end{aligned}
$$

where $\Delta m(\vec{r}, t) \equiv m(\vec{r}, t)-m^{(0)}$ is the excess spin density following spin injection, $m^{(0)}=n_{\uparrow}^{(0)}-n_{\downarrow}^{(0)}$ and $m(\vec{r}, t)$ $=n_{\uparrow}(\vec{r}, t)-n_{\downarrow}(\vec{r}, t)$ is the net spin density at point $\vec{r}$ and time $t$. In Eq. (45), $\tau_{s}=\left(1 / \tau_{s f, \uparrow}+1 / \tau_{s f, \downarrow}\right)^{-1}$ is the spinrelaxation time, which is very long, 4,12

$$
\begin{gathered}
\widetilde{D}_{\alpha}=D_{\alpha \alpha}-D_{\alpha \bar{\alpha}}, \\
\tilde{\mu}_{\alpha}=\mu_{\alpha \alpha}+\mu_{\alpha \bar{\alpha}},
\end{gathered}
$$

$\vec{E}$ is an externally applied electric field, and the matrix $\mu_{\alpha \beta}$ is defined as

$$
\begin{aligned}
e \mu_{\alpha \beta} & \equiv \frac{\partial \sigma_{\alpha \beta}}{\partial n_{\alpha}}-\frac{\partial \sigma_{\alpha \beta}}{\partial n_{\alpha}^{-}} \\
& = \pm 2 \frac{\partial \sigma_{\alpha \beta}}{\partial m}, \quad \text { plus if } \alpha=\uparrow, \text { minus otherwise. }
\end{aligned}
$$

This is a generalization of the familiar relation between mobility and conductivity: it takes into account the dependence of the mobility $\mu_{\alpha \beta}$ on both spin-density components. The second term in Eq. (48) accounts for the reduction of the mobility in the $\alpha$ channel due to the drag of the $\alpha$ spin population on the $\bar{\alpha}$ population.

The electrostatic field has the same sign for both spin components, while the density gradients have opposite signs [see Eq. (44)]: as a consequence the mobilities enter Eq. (45) as a spin symmetric combination [Eq. (47)] while the diffusion constants are in a spin-antisymmetric combination [Eq. (46)].

If we consider the linear regime-i.e., we neglect terms of the order of $\left(\nabla n_{\alpha}\right)^{2}-$ Eq. (48) reduces to the more familiar

$$
\sigma_{\alpha \beta}=e n_{\alpha} \mu_{\alpha \beta}
$$

and Eq. (45) reduces to the expression calculated in Ref. 7, i.e.,

$\frac{\partial \Delta m(\vec{r}, t)}{\partial t}=-\frac{\Delta m(\vec{r}, t)}{\tau_{s}}+D_{s} \nabla^{2} \Delta m(\vec{r}, t)+\mu_{s} \vec{E} \cdot \vec{\nabla} \Delta m(\vec{r}, t)$,

where

$$
\begin{gathered}
\mu_{s}=\frac{\sum_{\alpha} \tilde{\sigma}_{\alpha}^{-} \tilde{\mu}_{\alpha}}{\sum_{\alpha} \tilde{\sigma}_{\alpha}} \\
=\frac{1}{e} \frac{n}{n_{\uparrow} n_{\downarrow}} \frac{1}{\sum_{\alpha}\left(1 / \tilde{\sigma}_{\alpha}\right)}
\end{gathered}
$$

and

$$
\begin{aligned}
D_{s}= & \frac{\sum_{\alpha} \tilde{\sigma}_{\alpha}^{-} \tilde{D}_{\alpha}}{\sum_{\alpha} \tilde{\sigma}_{\alpha}} \\
= & \frac{S}{e^{2}} \frac{4}{\sum_{\alpha} \tilde{\rho}_{\alpha}}=\frac{k_{B} T}{e^{2} n} \cdot \frac{S}{S_{c}} \cdot \frac{1}{\left[\left(n_{\uparrow} n_{\downarrow} / n^{2}\right)\left(\rho_{D \uparrow}+\rho_{D \downarrow}\right)-\rho_{\uparrow \downarrow}\right]},
\end{aligned}
$$


are the effective mobility and diffusion constants. ${ }^{22}$ Here $\tilde{\rho}_{\alpha} \equiv \rho_{\alpha \alpha}-\rho_{\alpha \bar{\alpha}}, S_{c}$ is given by Eq. (37), $\rho_{D \alpha}=m^{*} / n e^{2} \tau_{\alpha}$ is the ordinary Drude resistivity associated with the $\alpha$ spin channel, and $\rho_{\uparrow \downarrow}$ - a negative number —is the spin-drag transresistivity discussed in Sec. III.

We underline that in reality the range of validity of Eq. (51) extends beyond the linear approximation into the classical regime, i.e., to high carrier densities or high temperatures, since in that regime the relationship between density and conductivity is linear. The solution of Eq. (51), with the initial condition $\Delta m(\vec{r}, 0)=\Delta M \delta(\vec{r})$, has the form of a Gaussian packet that drifts under the effect of the electric field $\vec{E}$ with a pace determined by $\mu_{s}$, and spreads in time at a rate determined by $D_{s}{ }^{7}$ The mobility and diffusion constants of electron-hole packets of similar shape can be measured through the Haynes-Shockley experiment: ${ }^{23}$ a similar experiment can then determine $\mu_{s}$ and $D_{s}$ independently.

We stress that Eqs. (52) - (55) apply to the general case in which the scattering times for the two spin components $\tau_{\alpha}$ 's may differ. Equations (52) and (54) show that the mobility and the diffusion constants of the packet are weighted averages of, respectively, the mobilities $\tilde{\mu}_{\alpha}$ and diffusion constants $\widetilde{D}_{\alpha}$ of the two spin channels, the weight being the conductivity $\tilde{\sigma}_{\bar{\alpha}}$ of the opposite channel. This is due to the local charge neutrality constraint Eq. (44), that forces the two components of the disturbance to travel together, so that the conductivity of each spin channel is strongly influenced by the motion of the disturbance in the other channel. In the noninteracting limit Eqs. (52) and (54) reduce to the expressions presented in Ref. 5.

Equation (53) can be seen as the generalization to a spin packet of the ordinary relationship between mobility and conductivity, while Eq. (55) is the corresponding generalization of the Einstein relation. Notice that, because the up- and down-spin components diffuse in opposite directions, friction arises between them, implying that the spin transresistivity enters Eq. (55) to decrease the packet diffusion. Moreover the spin stiffness $S$ is reduced by Coulomb interactions (see Fig. 4), and so it will be the rate of diffusion of the spin packet. It is interesting to notice that the presence of $\rho_{\uparrow \downarrow}$ in Eq. (55) represents an intrinsic mechanism by which diffusion is regulated (i.e., remains finite) even when the system is very pure and $\rho_{D \alpha} \rightarrow 0$. To derive the last expression in Eq. (55), we made use explicitly of the structure of the matrix $\rho_{\alpha \beta}$ discussed in Sec. II B.

If $\tau_{\uparrow}=\tau_{\downarrow}=\tau_{D}$, the expression of $\mu_{s}$ and $D_{s}$ is simplified, and we obtain ${ }^{7}$

$$
D_{s}=\frac{\mu_{s} k_{B} T}{e} \frac{S}{S_{c}} \frac{1}{1-\rho_{\uparrow \downarrow} / \rho_{D}},
$$

where $\rho_{D}=m^{*} / n e^{2} \tau_{D}$ is now the ordinary Drude resistivity, and $\mu_{s}=e \tau_{D} / m^{*}$, which shows that, under the assumption that up-spin and down-spin electrons have equal mobilities, the mobility of the packet coincides with the ordinary homogeneous mobility.

\section{EXPERIMENTAL OBSERVATION OF INTERACTION EFFECTS IN SPIN-POLARIZED TRANSPORT}

\section{A. Direct observation of spin Coulomb drag}

In Ref. 6 we proposed an experiment aimed at detecting the effect of the spin Coulomb drag and measuring the spin transresistivity. We described the experiment as it could be done on metals, but the same scheme could be applied to semiconductors (in which the drag effect is larger), provided that an appropriate method of injecting spin current is used.

The central idea is to use a paramagnetic metal film of thickness $L \ll \delta_{s}$ sandwiched between two ferromagnets polarized in the same direction. If a battery is connected to the ferromagnets, it will induce a spin-polarized current ${ }^{12}$ from the first ferromagnet ("injector") through the paramagnet and toward the second ferromagnet ("receiver"). ${ }^{24}$ If the injector and receiver are chosen to be half metals, i.e., they have only electron states of spin $\uparrow$ at the Fermi level, the circuit will behave as an open circuit for spin $\downarrow$ electrons. These electrons, due to the spin Coulomb drag effect only, will accumulate in the direction of the receiver creating a gradient in the spin $\downarrow$ density. As the stationary state is approached, the resulting electrochemical potential will exactly compensate the spin-drag force. By measuring this built-up electrochemical potential due to spin $\downarrow$ electrons, it will be possible to have a direct measure of the spin transresistivity. ${ }^{6}$

As shown by our calculations, we expect, in metals, a resistivity of the order of $10^{-2} \mu \Omega \mathrm{cm}$ proportional to $T^{2}$ (degenerate regime), while in semiconductors a resistivity as high as $10^{-2}-10^{-3} \Omega \mathrm{cm}$ for $T \approx T_{F}$.

\section{B. Haynes-Shockley experiment}

The Haynes-Shockley experiment ${ }^{23}$ demonstrated the drift and diffusion of minority carriers in a doped semiconductor. The experiment allows a direct and independent measure of the minority carrier diffusion and mobility coefficients. After a pulse of excess carriers is created at some point in the semiconductor, it drifts under the action of an electric field, for a known distance $L$, after which it is monitored. By measuring the drift time and the width of the packet, it is then possible to compute both the mobility and the diffusion constant of the packet, which coincide with those of the minority carriers.

The experiments of Ref. 4 can be seen as a HaynesShockley-type experiment, since they are based on a direct monitoring in space and time of unipolar spin packets. In these experiments, Kikkawa and Awschalom were able to measure independently the diffusion and the mobility of the spin packets. In the next section we are going to compare our theoretical results with their experimental findings.

\section{PARAMAGNETIC SEMICONDUCTORS AND SPONTANEOUS FERROMAGNETIC TRANSITION}

We will now focus on the results of our calculations for the diffusion constant of the spin packet in the paramagnetic regime $\left(n_{\uparrow}=n_{\downarrow}\right)$. In order to single out the interaction contribution to $D_{s}$, in Fig. 5 we plot the ratio $D_{s} / D_{n i}$, where 


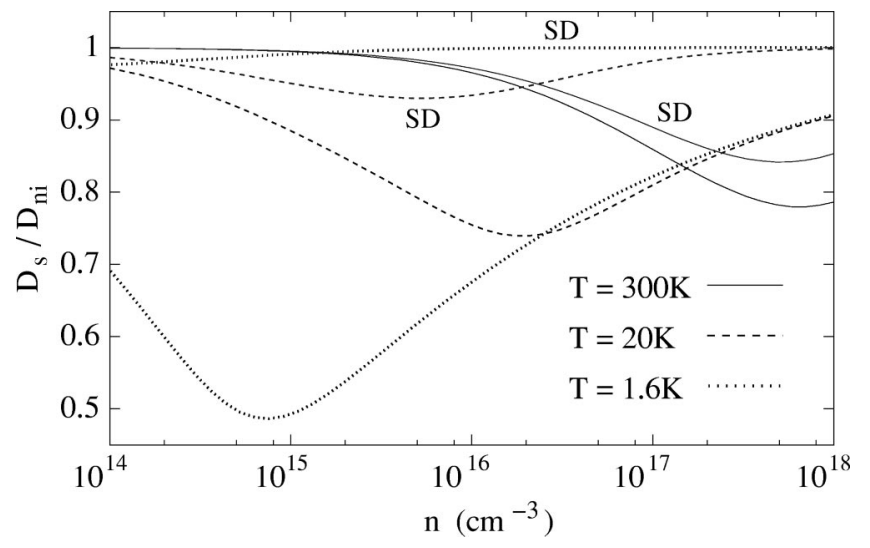

FIG. 5. The interacting diffusion constant of a spin packet $D_{s}$ rescaled by its noninteracting approximation $D_{n i}$ vs density for different temperatures: solid lines correspond to $T=300 \mathrm{~K}$, dashed lines to $T=20 \mathrm{~K}$ and dotted lines to $T=1.6 \mathrm{~K}$. For each temperature, we plot also the curve obtained considering interactions only through the spin Coulomb drag effect (labeled SD). In all the calculations the dielectric constant of the semiconductor is $\epsilon=12$ and the mobility is $\mu=3 \times 10^{3} \mathrm{~cm}^{2} / \mathrm{V} \mathrm{s}$.

$D_{s}$ represents the fully interacting calculation according to Eq. (56) and $D_{n i}$ is the noninteracting diffusion constant, as in Ref. 5. The figure shows results for $n$-doped GaAs, at three different temperatures and for densities relevant to the experiments of Ref. 4. As expected, we see that $D_{s} / D_{n i}<1$ always; moreover the interaction correction can be as high as $50 \%$. The solid lines correspond to the calculations performed at a temperature $T=300 \mathrm{~K}$, the dashed lines to $T=20 \mathrm{~K}$, and the dotted lines to $T=1.6 \mathrm{~K}$. The curves marked with "SD" correspond to the case in which interactions in $D_{s}$ are taken into account only through the spin Coulomb drag effect. The figure shows clearly that at low temperatures the most important many-body contribution to the diffusion is due to the softening of the spin stiffness, while, already at $20 \mathrm{~K}$ the spin-drag contribution becomes relevant, to represent most of the interaction effects at room temperature. Despite the significant reduction due to the interaction correction, $D_{s}$ remains still considerably larger than $D_{c}{ }^{7}{ }^{7}$ consistent with experimental observations. ${ }^{4}$

We see that $D_{s} / D_{n i} \rightarrow 1$ for high densities. This is due to the enhancement of screening in this regime, so that the particles tend to behave as noninteracting ones. In the highdensity regime $S \approx S_{n i}$, as can be seen in Fig. 4. If the temperature is high enough, the spin drag still reduces the diffusion constant by a sizable amount, but eventually, even this contribution disappears with increasing density, and $D_{s}$ $\rightarrow D_{n i}$. At low density, the system enters the nondegenerate regime $T \gg T_{F}(n)$, so that both $D_{s} \rightarrow D_{c}$ and $D_{n i} \rightarrow D_{c}$, where $D_{c}=\mu_{s} k_{B} T / e$ is the classical noninteracting diffusion constant. However, in the noninteracting theory ${ }^{5}$ the nondegenerate limit $D / D_{c} \rightarrow 1$ is approached from above, while, due to the spin Coulomb drag, $D_{s} / D_{c} \rightarrow 1$ from below always. ${ }^{7}$

We want to stress that $D_{s}$ also displays a marked dependence on the sample mobility that affects the diffusion constant through Drude resistivity $\rho_{D}$ [see Eq. (56)]. As we al- ready underlined in Sec. III A, the higher the mobility, the more important becomes the ratio $\left|\rho_{\uparrow \downarrow}\right| / \rho_{D}$, that can reach values even greater than 1 (see Figs. 2 and 3). The diffusion constant in these cases is then regulated by the spin-drag effect that cannot be neglected.

By looking at the behavior of the spin stiffness (Fig. 4) and at Eqs. (55) and (56), it is clear that, when the electron gas undergoes a ferromagnetic transition, $D_{s}$ will display large variations. ${ }^{7}$ In fact $S$ (and so $D_{s}$ ) vanishes at the transition temperature $T_{c}$ and increases sharply as the system settles in the fully polarized state. In the case of intrinsic ferromagnetism, the critical behavior of $D_{s}$ is completely due to Coulomb interactions among carriers: in this regime of extremely low carrier density in fact, even when $T \approx T_{c}$, $D_{n i} \approx D_{c}$. On the other side, as the system fully polarizes $n_{\downarrow} \rightarrow 0, \rho_{\downarrow \uparrow} / \rho_{D} \rightarrow 0$, and $S / S_{c} \rightarrow 1$, as demonstrated in Sec. IV B, so that $D_{s}$ reduces to the diffusion constant of carriers of minority orientation (which are nondegenerate), i.e., to the classical value $D_{c}=k_{B} T \mu / e^{7}$

As a final remark, we would like to underline that semiconductors doped with magnetic impurities (for example, $\mathrm{Mn}$ ) can undergo a ferromagnetic transition at rather high carrier densities, ${ }^{25,26} n \sim 10^{20} \mathrm{~cm}^{-3}$ for $(\mathrm{Ga}, \mathrm{Mn}) \mathrm{As}$, and temperatures $T<T_{c} \sim 110 \mathrm{~K} .{ }^{25}$ Our theory on the dynamics of a spin packet can be extended to these systems with similar results. ${ }^{7}$ This extension will not be pursued here.

\section{CONCLUSIONS}

In this paper, we have tried to demonstrate the importance of many-body effects in spin-polarized transport. We have discussed in detail the spin Coulomb drag effect, an intrinsic source of friction in spin transport that can limit spin currents even in the purest materials. We have worked out the behavior of the spin transresistivity $\rho_{\uparrow \downarrow}$ in different physical regimes and shown that it ranges from $10^{-8} \Omega \mathrm{cm}$ in metals to $10^{-3}-10^{-2} \Omega \mathrm{cm}$ in semiconductors. Moreover, the ratio $\rho_{\uparrow \downarrow} / \rho_{D}$, which is only a few percents in metals, becomes comparable to, or even larger than unity in semiconductors. We hope that an experimental group will soon take up the challenge of measuring the spin transresistivity, for example, through the experiment we suggest, in order to confirm the theory.

We have also demonstrated the importance of including Coulomb interactions in a quantitative theory of spin diffusion, and shown that a measure of $D_{s}$ for a unipolar spin packet would be a sensitive probe of many-body effects such as the spin Coulomb drag and the Coulomb enhancement of the spin susceptibility.

Finally we have discussed the behavior of $D_{s}$ at and below a spontaneous ferromagnetic ordering transition and found that $D_{s}$ exhibits a critical behavior.

\section{ACKNOWLEDGMENT}

We gratefully acknowledge support from NSF grant No. DMR-0074959. 
${ }^{1}$ G. A. Prinz, Phys. Today 48(4), 58 (1995); G. A. Prinz, Science 282, 1660 (1998); D. D. Awschalom and J. M. Kikkawa, Phys. Today 52(6), 33 (1999).

${ }^{2}$ D. Loss and D. P. DiVincenzo, Phys. Rev. A 57, 120 (1998); B. Kane, Nature (London) 393, 139 (1998).

${ }^{3}$ J. M. Kikkawa and D. D. Awschalom, Phys. Rev. Lett. 80, 4313 (1998); J. M. Kikkawa, I. P. Smorchkova, N. Samarth, and D. D. Awschalom, Science 277, 1284 (1997).

${ }^{4}$ J. M. Kikkawa and D. D. Awschalom, Nature (London) 397, 139 (1999).

${ }^{5}$ Michael E. Flatté and Jeff M. Byers, Phys. Rev. Lett. 84, 4220 (2000).

${ }^{6}$ I. D'Amico and G. Vignale, Phys. Rev. B 62, 4853 (2000).

${ }^{7}$ I. D’Amico and G. Vignale, Europhys. Lett. 55, 566 (2001).

${ }^{8}$ D. Ceperley and B. J. Alder, Phys. Rev. Lett. 45, 566 (1980); Int. J. Quantum Chem. 16, 49 (1982).

${ }^{9}$ G. Ortiz, M. Harris, and P. Ballone, Phys. Rev. Lett. 82, 5317 (1999).

${ }^{10}$ For simplicity, we limit ourselves in this paper to either the paramagnetic case or the ferromagnetic case with collinear spins.

${ }^{11}$ We assume that impurities have a negligible effect on the thermodynamic functions of the electron gas.

${ }^{12}$ M. Johnson and R. H. Silsbee, Phys. Rev. Lett. 55, 1790 (1985); Phys. Rev. B 37, 5312 (1988); 37, 5326 (1988).

${ }^{13}$ D. Pines and P. Nozieres, The Theory of Quantum Liquids (W. A. Benjamin, Inc., New York, 1966).

${ }^{14}$ N. D. Mermin, Phys. Rev. B 1, 2362 (1970).

${ }^{15}$ For a recent review of the theoretical and experimental situation on Coulomb drag, see A. G. Rojo, J. Phys.: Condens. Matter 11, R31 (1999), and references therein.

${ }^{16}$ Coulomb drag effects between different carrier species in semiconductors have been studied by several authors, e.g., M. Combescot and R. Combescot, Phys. Rev. B 35, 7986 (1987); W. Hänsch and G. D. Mahan, J. Phys. Chem. Solids 44, 663 (1983);
T. P. McLean and E. G. S. Paige, ibid. 16, 220 (1960). No previous work dealt, to the best of our knowledge, with the spin degrees of freedom.

${ }^{17}$ The sign of $\rho_{\uparrow \downarrow}$ is negative because the electrochemical field $\mathbf{E}_{\downarrow}$ must oppose the drag force which pulls the spin-down electrons in the direction of motion of the spin-up electrons.

${ }^{18}$ S. Tanaka and S. Ichimaru, Phys. Rev. B 39, 1036 (1989).

${ }^{19}$ Historically, the first prediction of ferromagnetism in the homogeneous interacting electron gas dates back to Bloch, who predicts a first-order transition at $r_{s}=5.45$ within the Hartree-Fock approximation. Later Monte Carlo studies (Ref. 8) placed the transition at a much higher value of $r_{s} \sim 75$, where the liquid ferromagnetic phase competes with the Wigner crystal phase. More recent Monte Carlo studies (Ref. 9) find what appears to be a continuous transition from the paramagnetic to the ferromagnetic fluid at smaller values of $r_{s} \sim 20 \pm 5$, in qualitative agreement with the prediction of Ref. 18.

${ }^{20}$ K. Huang, Statistical Machanics (Wiley and Sons, New York, 1987).

${ }^{21}$ L. D. Landau and E. M. Lifshitz, Course of Theoretical Physics (Pergamon Press, New York, 1993), Vol. 5, Sec. 80.

${ }^{22}$ In the spirit of the linear-response approximation these constants should be evaluated at the underlying equilibrium density of the electron gas.

${ }^{23}$ See, for example, B. G. Streetman, Solid State Electronic Devices (Prentice Hall, Englewood Cliffs, N.J., 1980).

${ }^{24}$ Ideally, one would like to use an injector that is closely matched in impedance to the paramagnet, so that all the injected current goes into an average drift velocity of the up-spin component and none is "lost" in the form of a diffusion current.

${ }^{25}$ H. Ohno, Science 281, 951 (1998).

${ }^{26}$ B. König, U. Zehnder, D. R. Yakovlev, W. Ossau, T. Gerhard, M. Keim, A. Waag, and A. Landwehr, Phys. Rev. B 60, 2653 (1999). 